\title{
SignA, el geoportal del IGN de España: explotando lo mejor de las IDE y los SIG
}

\author{
Hugo Potti Manjavacas* \\ Celia Sevilla Sánchez ${ }^{*}$ \\ Miguel Villalón Esquinas* \\ Jaime Sánchez Fanjul ${ }^{*}$
}

Recibido el 16 de enero de 2017; aceptado el 13 de junio de 2017

\begin{abstract}
SignA, the National Geographic Information System of Spain, has been a strategic project of the National Geographic Information Centre (CNIG) and the National Geographic Institute (IGN) of Spain, which aims the IGN data and services integration within a Geographic Information System (GIS), for its management, analyse and query through the Internet. This implies the development of an own versatile, interoperable and efficient geoportal.

SignA geoportal was opened to public on December 2010 and it is about to open the fourth version, combining the best features of the Spatial Data Infrastructures (SDI) and GIS technologies, allowing users to access data and services in an easy and transparent way, without special technical knowledge. After 7 years, the geoportal is growing every day its importance and usage onto the geomatics community. Currently, receives an average of 19,000 visits per month, being a tool of daily use for many of our users and for many applications.

This article will be a brief review of the main features of the geoportal and the changes made to improve usability over the versions.

Key words: Geographic Data, GIS, SDI, SignA, IGN, CNIG, INSPIRE, OGC.
\end{abstract}

* Centro Nacional de Información Geográfica-Instituto Geográfico Nacional de España (CNIG-IGN), Área de proyectos internacionales, calle General Ibañez Ibero 3, 28003 Madrid, España, correos electrónicos: signa@ign.es, hpottil@fomento.es, cssanchez@fomento.es, miguel.villalon@cnig.es, jaime.sanchez@cnig.es 


\section{Resumen}

El Sistema de Información Geográfica Nacional (SignA) es un proyecto estratégico del Instituto Geográfico Nacional de España (IGN) que tiene como finalidad, la integración de los datos y servicios del IGN en un Sistema de Información Geográfica (SIG), para su gestión, análisis y consulta, a través de Internet, lo que a su vez implica el desarrollo de un geoportal propio versátil, interoperable y eficiente.

El geoportal del SignA, se abrió al público en diciembre de 2010 y próximamente sacará la tercera versión, integrando lo mejor de los mundos SIG e IDE (Infraestructura de Datos Espaciales) en una única herramienta, permitiendo a los usuarios acceder a datos y servicios de manera fácil y transparente, sin requerir de conocimientos técnicos concretos. Tras siete años, ha ido aumentando la demanda del geoportal, actualmente recibe una media de 19,000 visitas al mes, y es una herramienta de uso de diario de muchos de nuestros usuarios para multitud de aplicaciones.

En este artículo se da un breve repaso a las características del portal, resaltando las mejoras de usabilidad realizadas a lo largo de las diferentes versiones.

Palabras clave: Datos geográficos, Sistema de Información Geográfica, Infraestructura de Datos Espaciales, SIGNA, IGN, CNIG, INSPIRE, OGC.

\section{Resumo}

O Sistema de Informação Geográfica Nacional (SignA) é um projeto estratégico do Instituto Geográfico Nacional da Espanha (IGN) que tem como finalidade, a integração dos dados e serviços do IGN em um Sistema de Informação Geográfica (SIG), para sua gestão, análises e consultas, através de Internet, o que por sua vez implica no desenvolvimento de um geoportal próprio e versátil, interoperável e eficiente.

O geoportal do SignA, se abriu ao público em dezembro de 2010 e em breve será disponibilizada a terceira versão, integrando o melhor dos mundos SIG e IDE (Infraestructura de Datos Espaciales) em uma única ferramenta, permitindo aos usuários acessar a dados e serviços de maneira fácil e transparente, sem precisar de conhecimentos técnicos concretos. Nos últimos 7 anos, tem aumentando a demanda de acesso ao geoportal, onde atualmente recebe uma média de 19.000 visitas ao mês, sendo uma ferramenta de uso de diário de muitos nossos usuários para muitas aplicações.

Neste artigo se faz um breve repasse das características do portal, ressaltando as melhoras de usabilidade realizadas ao longo de diferentes versões.

Palavras chave: Dados geográficos, Sistema de Informação Geográfica, Infraestrutura de Dados Espaciais, SIGNA, IGN, CNIG, INSPIRE, OGC. 


\section{Introducción}

El Instituto Geográfico Nacional de España (IGN), ha estado trabajando desde la década de los setenta con Sistemas de Información Geográfica (SIG) siendo estos el resultado de la aplicación de las Tecnologías de Información (TI) a la gestión y análisis de información geográfica, con la capacidad de gestionar grandes volúmenes de datos a gran velocidad y con repetitividad.

Con la llegada de los avances legislativos en materia de Información Geográfica y su consecuencia tecnológica en forma de Infraestructuras de Datos Espaciales (IDE), se permite la interoperabilidad de diferentes sistemas a través de Internet, gracias al cumplimiento de una serie de normas y especificaciones pactadas, y a la existencia de unos sistemas que contienen datos georreferenciados distribuidos, metadatos y servicios. Esto lleva a disponer de dos mundos, SIG e IDE, potencialmente complementarios, pero no tan fáciles de mezclar: la potencia de las herramientas que proporcionan los SIG y la ingente cantidad de datos que se ponen a disposición del usuario a través de las IDE.

En ese momento nació el Sistema de Información Geográfica Nacional (SignA), un proyecto estratégico del IGN que tiene como finalidad la integración de los datos y servicios interoperables de información geográfica del IGN en un SIG, que además está disponible en Internet para todo tipo de usuarios a través de un geoportal, cuya utilización solo requiere un navegador web común.

El geoportal del SignA se puso en marcha a finales del año 2010 y desde entonces permite la visualización, impresión, consulta y análisis de los datos geográficos oficiales del IGN (Sevilla, Abad y Villalón, 2011; Sevilla, Potti, Abad, Rodríguez, 2010), usando la propia base de datos y los servicios web del IGN que cumplen estándares del Open Geospatial Consortium (OGC), así como los miles de servicios estándar creados y mantenidos por otros organismos o empresas.

Al integrar los datos geográficos oficiales del IGN con otras fuentes de datos a través de servicios web estándar, el SignA se convierte en una potente herramienta para ciudadanos, empresas privadas, universidades y organismos públicos que necesitan visualizar, analizar y comparar información geográfica de muy diversa índole.

A lo largo del presente artículo se esbozan las principales características de SignA, las mejoras en usabilidad hechas a lo largo de las versiones, la elección de los datos y servicios a mostrar, una descripción general del cliente y una visión de los estados presente y futuro del proyecto.

\section{SignA, el punto de acceso privilegiado al nodo IDE del IGN}

En el Capítulo I de la Ley 14/2010, de 5 de julio, sobre las Infraestructuras y los Servicios de Información Geográfica en España (LISIGE) que transpone la directiva europea INSPIRE se definen: 


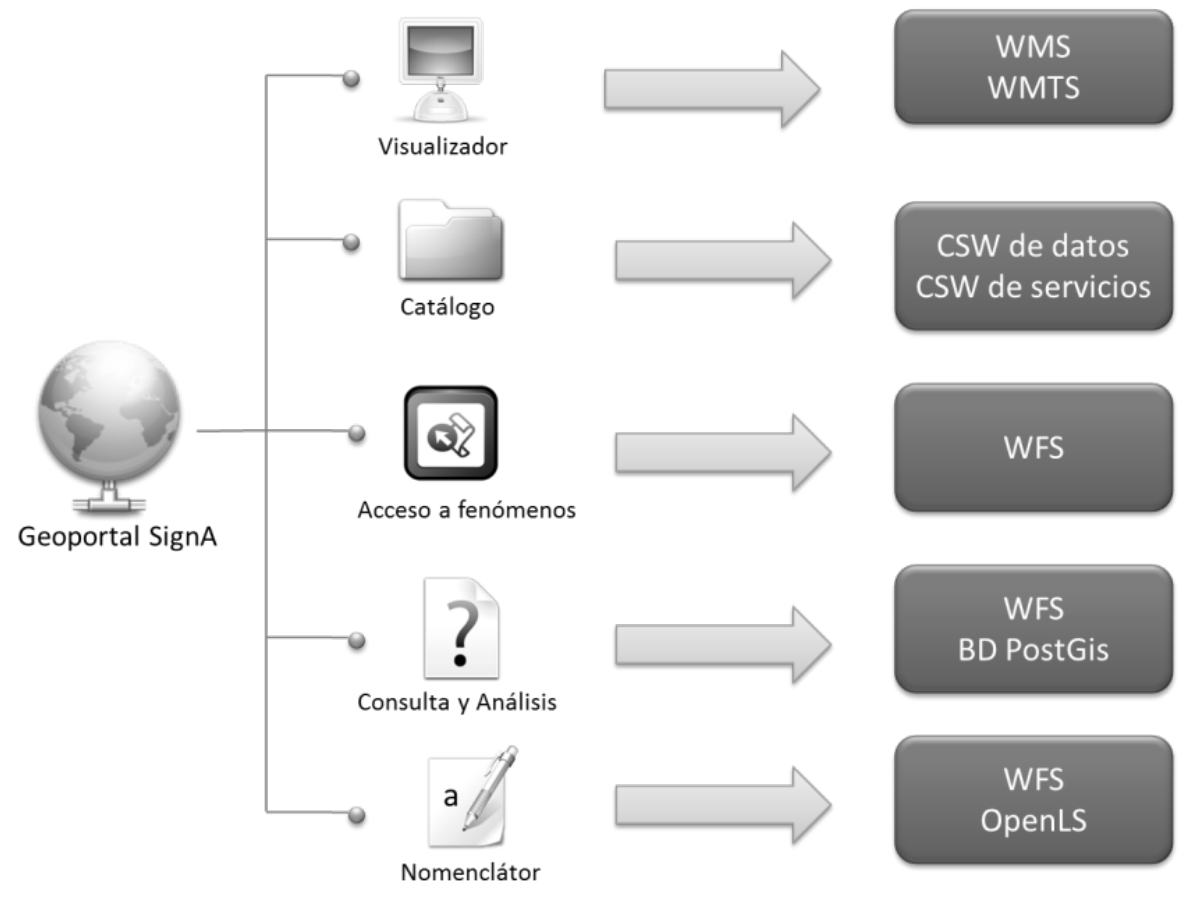

Figura 1. Estructura del contenido de SignA y su conexión a BD o servicios OGC.

- Geoportal: sitio Internet o equivalente que proporciona acceso a servicios interoperables de información geográfica de varios órganos, organismos o entidades de una o varias Administraciones Públicas, e incorpora al menos un servicio que permita buscar y conocer los datos y servicios geográficos accesibles a través de él.

- Nodo de Infraestructura de Información Geográfica: conjunto de servicios interoperables de información geográfica accesibles, a través de Internet, por la acción de un órgano, organismo o entidad de las Administraciones Públicas.

Teniendo en cuenta lo expuesto anteriormente, podemos decir que SignA es un geoportal que, por estar implementado por el IGN y por integrar en un único entorno la explotación SIG de las bases de datos del IGN y de sus servicios interoperables, constituye un punto de acceso privilegiado al nodo IDE del IGN.

El geoportal del SignA muestra todos y cada uno de los servicios OGC que el IGN genera como Servicios Web estándar de: Visualización de Mapas (WMS), Visualización de Mapas Teselados (WMTS), Descarga de Objetos Geográficos (WFS), Catálogo (CSW) y Coberturas (WCS), además de una selección de los datos 
que componen la base de datos interna del SignA. Además, en la funcionalidad de entrada de nuevas fuentes de datos, enlaza al Directorio de Servicios de la IDEE, lo que permite cargar los servicios web estándar registrados en él y que están clasificados en nacionales, regionales y locales: más de 2,000 servicios WMS, más de 425 WFS, más de 40 CSW, etc.

De esta manera, SignA se constituye como un geoportal IDE, que aprovecha y optimiza las posibilidades de la interoperabilidad y normalización proporcionada por un nodo IDE, con el valor añadido del análisis SIG. Bajo estas premisas ambos mundos se combinan de manera armónica a través de un solo acceso.

\section{Datos y servicios de SignA}

A grandes rasgos, SignA analiza datos de dos maneras diferenciadas: mediante la conexión directa a su propia base de datos almacenada en PostGIS y a través de la conexión a múltiples tipos de servicios OGC, ya que es un cliente ligero de servicios estándar.

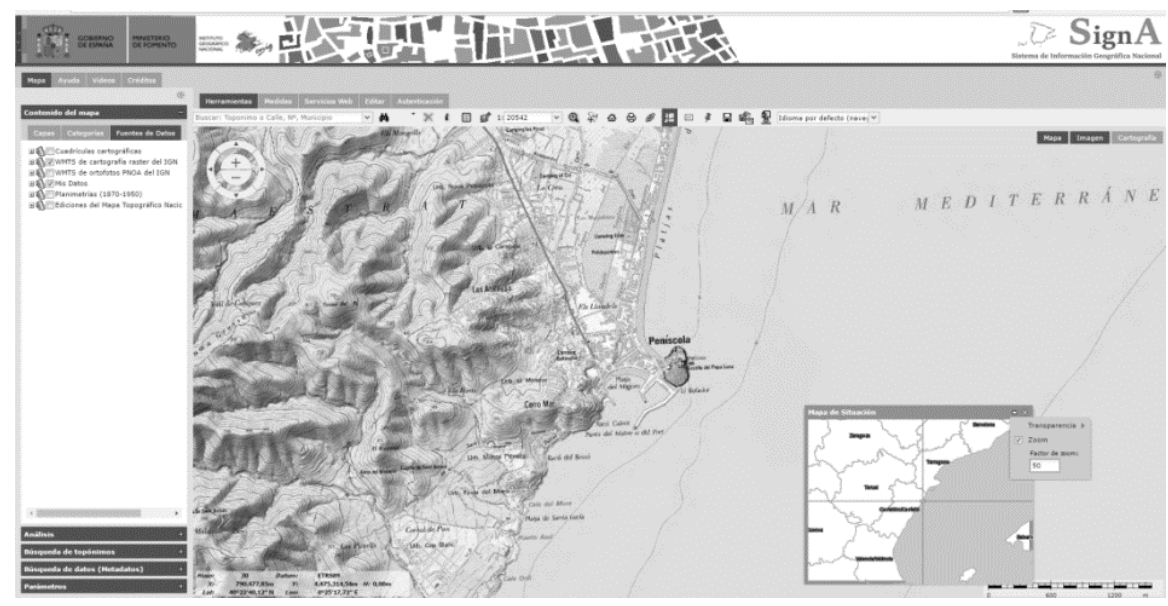

Figura 2. Captura de pantalla de SignA.

\section{Base de datos SignA}

La base de datos del SignA se compone de datos geográficos y alfanuméricos procedentes de los distintos proyectos existentes en el IGN. Actualmente, se podría decir que la escala general de los datos almacenados es 1:100.000, pero se encuentran excepciones que permiten una correcta representación cartográfica a escalas mayores. Los datos que se han cargado en la base de datos, se han seleccionado de las diferentes fuentes existentes y se han adaptado para permitir su consulta mediante herramientas SIG, añadiendo atributos para enriquecer los resultados. En este 
sentido, se ha hecho una labor de traducción de los campos para que el usuario vea atributos textuales en lugar de códigos y así no tener que hacer uso del catálogo de datos. Por ejemplo, en las autopistas la calzada si es doble (02) o de un único sentido (03) se escribe literalmente para que el usuario lo entienda, aunque en la base de datos sigue con códigos para que las búsquedas sean más rápidas.

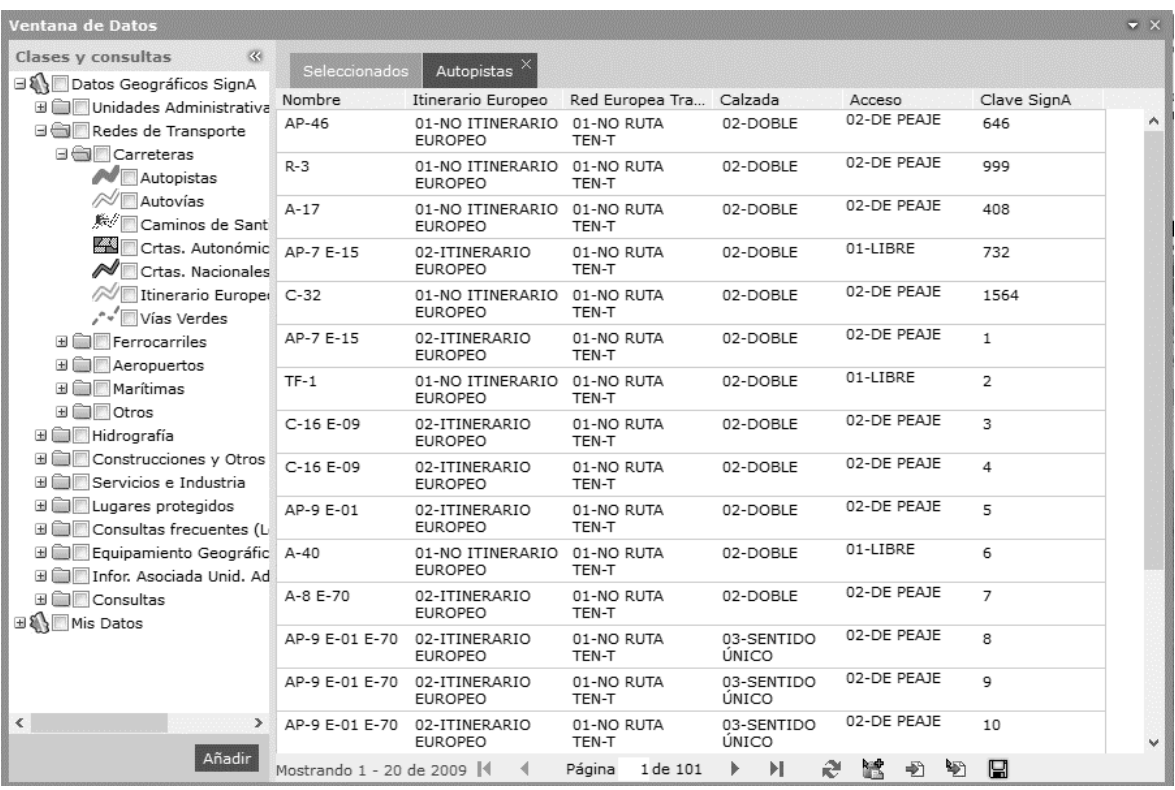

Figura 3. Vista de datos de la capa de autopistas.

Los proyectos de los que se han obtenido los datos son fundamentalmente: las Bases Topográficas Nacionales a escala 1:25.000 y 1/100.000 (BTN100, BTN25) y Euro Regional Map (ERM). Aunque en un futuro próximo se añadirán los de la Información Geográfica de Referencia (IGR) que se están produciendo a la mayor resolución disponible y por temáticas (hidrografía, red de transporte, entidades de población, usos del suelo, etc.).

Para organizar la información de manera jerárquica y que se encuentren de manera sencilla se ha seguido la clasificación por temas de INSPIRE:

- Unidades administrativas: comunidades autónomas, provincias, municipios, etc.

- Redes de Transporte: carreteras, ferrocarriles, aeropuertos, puertos, etc.

- Hidrografía: cursos de agua, embalses, lagunas, etc.

- Edificios: paradores, iglesias, castillos, monumentos, balnearios, parques de atracciones, etc. 
- Instalaciones de producción e industriales: centrales eléctricas, centrales nucleares, conducciones de combustible, etc.

- Lugares Protegidos: parques nacionales, parques naturales, reservas, etc.

- Sistema de cuadrículas geográficas: distribución de hojas MTN25 y MTN50.

- Direcciones.

En total se han cargado 117 clases de objeto geográfico, que conforman la base de datos del SignA y que se pueden visualizar, consultar y descargar. La conexión del geoportal a la base de datos es directa, lo que ayuda a un procesado más eficiente. Las consultas que se pueden hacer sobre estos datos son: consultas por atributos o alfanuméricas, consultas espaciales, consultas mixtas y áreas de influencia.

\section{Servicios OGC}

El IGN como organismo cartográfico nacional está compuesto por numerosos departamentos que producen un amplio y variado abanico de datos y servicios. Cada uno de estos departamentos está generando servicios publicados conforme a estándares que precisan de un lugar en el que se visualicen y analicen los datos geográficos. En enero de 2017, el IGN dispone de:

- 17 servicios de visualización de mapas o Web Map Service (WMS) y 6 Web Map Tiled Service (WMTS) que ofrecen, por ejemplo, datos geofísicos como son las anomalías de Bouguer, datos geodésicos como el geoide de España o datos cartográficos como las Bases Topográficas Nacionales, mapas topográficos, modelos digitales del terreno, ortofotos, imágenes de satélite, etc.

- 12 servicios de acceso a objetos geográficos o Web Feature Service (WFS) como por ejemplo las líneas límite, los vértices geodésicos o los servicios de nomenclátor.

- Un servicio de coberturas o Web Coverage Service (WCS) correspondiente a los modelos digitales del terreno del IGN.

- Un servicio procesamiento de información geográfica o Web Processing Service (WPS) donde por ejemplo se puede consultar la altura máxima y mínima de una zona o calcular la visibilidad entre dos puntos del terreno.

- Un servicio de catálogo o Catalogue Service Web (CSW) donde consultar los metadatos de datos y servicios del IGN.

- Un servicio de transformación de coordenadas o Web Coordinate Transformation Service (WCTS).

La dirección URL de estos servicios está disponible en <http://www.idee.es/ web/guest/directorio-de-servicios>. 
Para que los usuarios no tengan que acceder a la URL anterior y copiar y pegar la URL de los servicios que desean cargar, se hizo una mejora en la versión 1 para que el geoportal mostrara el directorio de servicios de la IDEE y que al pulsar en el árbol de servicios se pudieran cargar otros servicios OGC externos de manera sencilla. En la siguiente figura se puede ver el árbol de servicios web estándar que hay en España y que se extrae periódicamente de la web de la IDEE.

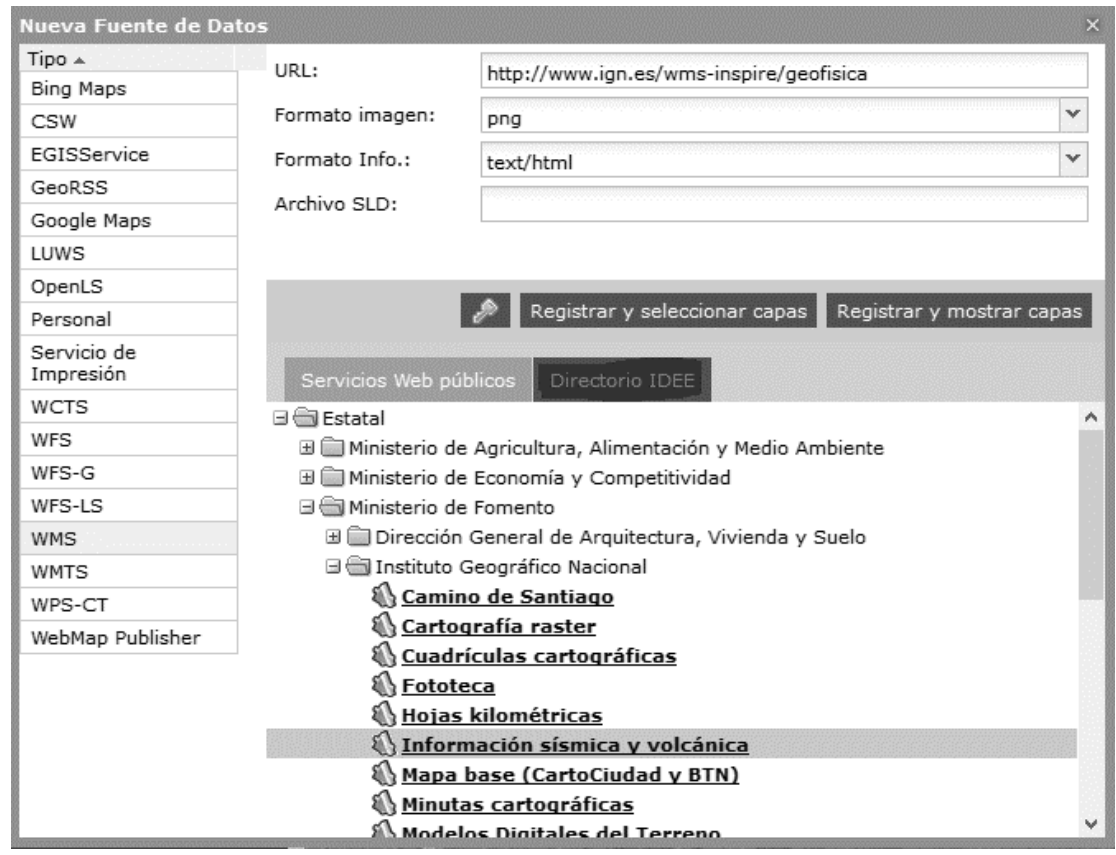

Figura 4. Carga de servicios web directa a partir de la URL del Directorio de la IDEE.

El proyecto SignA explota los servicios, pero no es responsable de su mantenimiento y no proporciona nuevos servicios, salvo alguna excepción como el servicio WMTS del mapa base de la aplicación.

En este sentido, el proyecto SignA fue pionero en la creación y explotación de servicios utilizando este nuevo estándar de publicación de mapas teselados, WMTS, publicado por OGC en abril de 2010.

En cada vista (mapa, imagen o cartografía) se dispone de una serie de servicios precargados para cada escala, así se permite que los usuarios «básicos» accedan a los servicios web estándar de manera sencilla. Además, se han configurado los servicios y la transparencia de las capas por escalas para que el usuario solo tenga que navegar. Además, se han añadido en cada vista capas desactivadas del IGN que normalmente piden los usuarios para que las puedan apagar o encender con un 
check box sin necesidad de conectarse a ningún servicio y cargarlo. Por ejemplo, en la vista inicial se ha precargado el servicio que muestra los terremotos de los últimos días porque se ha demostrado, por las estadísticas de acceso al geoportal, que cuando hay un terremoto suben las visitas. De esta manera los usuarios que entren al geoportal activan la capa sin necesidad de conocer qué servicio hay que cargar ni como cargarlo. Otro ejemplo, sería la vista de Cartografía que por defecto visualiza el servicio WMTS con la cartografía raster de España, pero el usuario, si lo desea, puede superponer el servicio WMTS con las ortoimágenes aéreas que ya tiene transparencia configurada para visualizar ambas capas conjuntamente. Además, al hacer zoom es posible activar otras capas como la rejilla con la distribución de hojas, que permite ver el nombre y número de hoja del MTN25 y MTN50; la primera edición del MTN50 que se empezó en 1875 y que permite analizar la evolución del territorio; o las minutas cartográficas originales. De manera similar, se ha hecho una configuración de capas para la vista de imagen que permite añadir ortofotos históricas y fotografías de vuelos antiguos.

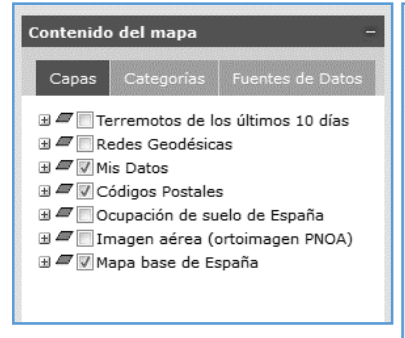

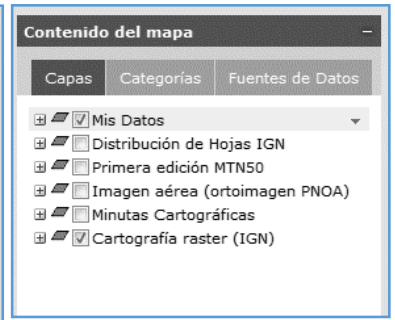

$\square$ Fototeca: Vuelo Ruíz de Alda

$\pm \approx$ Imagen aérea (PNOA más actual) $\boxplus \nabla \nabla$ Información Mosaico PNOA

Figura 5. Capas precargadas en el SignA en las vistas de mapa, imagen y cartografía.

También se ha desarrollado un Servicio de Localización basado en Identificadores Geográficos, a partir de las direcciones proporcionadas por el proyecto CartoCiudad y de los topónimos del Servicio del Nomenclátor Geográfico Básico del IGN. Este buscador, se ha implementado a través de un servicio Open Location Service (OpenLS) que mediante un único cuadro de texto permite localizar direcciones, entidades de población o municipios, siendo el propio cliente el encargado de buscar en una u otra base de datos. En todas las versiones hasta la v.4, como los usuarios tienden a escribir las direcciones introduciendo el carácter coma “,” para separar el número del portal o la entidad de población, se eligió este carácter para que el buscador automáticamente buscase en el servicio de Nomenclátor cuando no hubiese coma o en el de Cartociudad (callejero) al introducir la coma. Sin embargo, se ha mejorado en la versión 4 y la herramienta busca en ambos servicios y posteriormente, el usuario elige cuál se asemeja más a su búsqueda. 


\section{Funcionalidades de SignA}

El principal objetivo del proyecto ha sido el desarrollo de un portal web diseñado para todo tipo de usuarios, conforme a estándares (OGC, W3C), evitando el uso de plugins y con el objetivo de que funcione de una manera estable sobre los navegadores más populares y en las versiones más usadas.

Dos principales características que marcan su buen rendimiento son su comportamiento asíncrono y el estar basado esencialmente en servicios de mapas cacheados basados en el reciente estándar WMTS de OGC.

En cuanto a las funcionalidades del portal se podrían dividir en funcionalidades básicas, SIG e IDE (Sevilla et al., 2016). En este texto omitiremos las básicas para centrarnos en las SIG e IDE, no obstante, en la siguiente dirección se puede ver un Seminario Web celebrado a mediados de 2016 en el que se puede ver un resumen de $1 \mathrm{~h}$ de todas las funcionalidades de SignA < www.youtube.com/QwUn1g92qQI $>$.

\section{Funcionalidades IDE}

Las Infraestructuras de Datos Espaciales permiten la integración de datos y servicios procedentes de diferentes fuentes, de manera interoperable. La aplicación se ha diseñado para que los usuarios con conocimientos en IDE saquen el máximo potencial al portal al poder acceder a todo tipo de información de otros organismos mundiales, nacionales, regionales y locales. Las funcionalidades IDE que implementa SignA son:

- Solicitud de la información asociada a una entidad procedente de un servicio CWS (operación GetFeatureInfo).

- Carga e integración de diferentes servicios web estándar: WMS, WMTS, WFSG CSW, OpenLS, WFS, etc.

- Almacenamiento de los parámetros de conexión a servicios WMS con el estándar Web Map Context (WMC) para poder cargar la configuración del mapa actual en otros portales o aplicaciones de escritorio (como GvSIG).

- Búsqueda de datos y productos utilizando el servicio de catálogo CSW. La búsqueda se realiza a través de los metadatos conforme al Reglamento de Metadatos de la Directiva INSPIRE.

- Búsqueda de nombres geográficos para tener su localización y visualizar sus metadatos, a través de servicios WFS-G.

- Consultas temáticas, espaciales y áreas de influencia sobre los datos obtenidos de servicios WFS. 


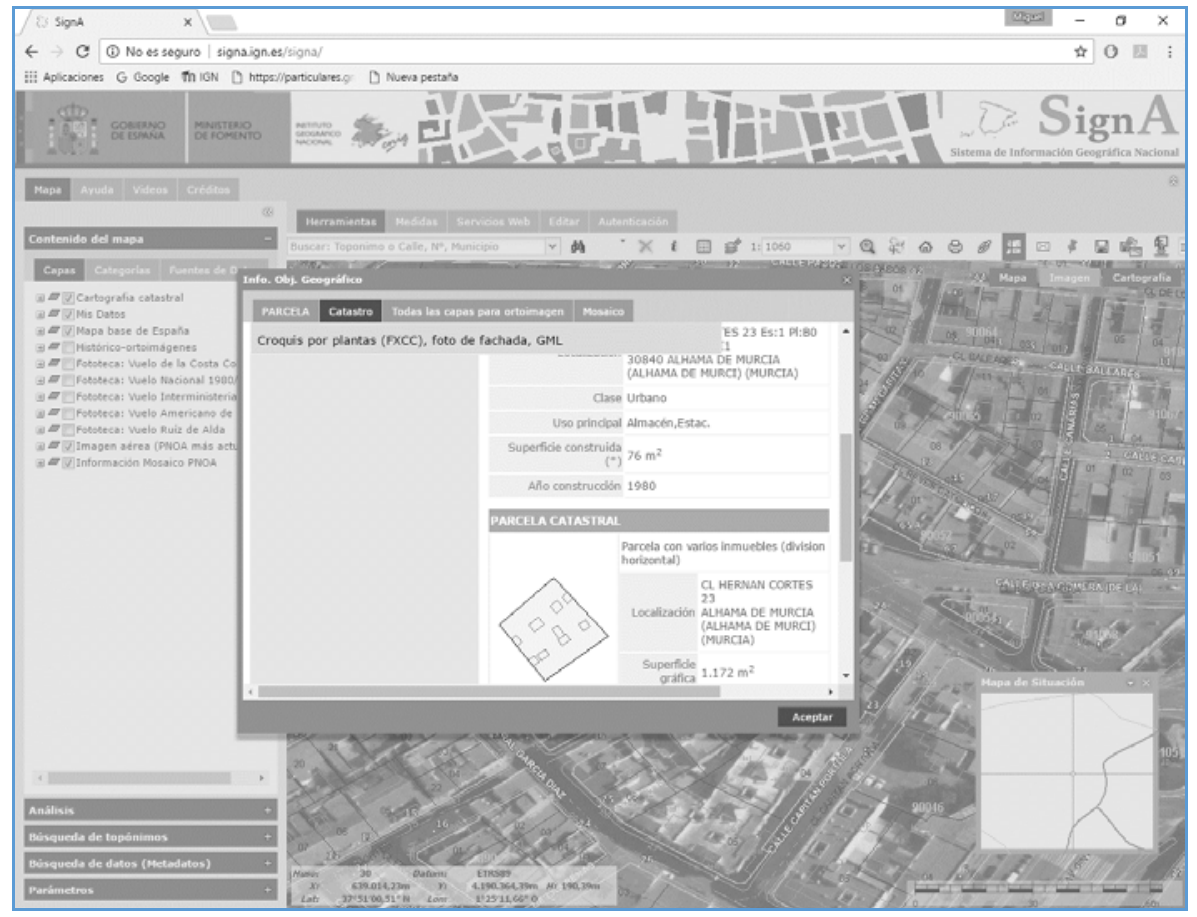

Figura 6. Información asociada al servicio de catastro mostrada por SignA.

\section{Funcionalidades SIG}

Los Sistemas de Información Geográfica son una potente herramienta que permite analizar la información geográfica y la toma de decisiones en función de los resultados obtenidos. La aplicación está en constante evolución, siendo nuestro principal objetivo conseguir que los usuarios con conocimientos en SIG sean capaces de hacer consultas en línea con los datos de la base de datos del SignA o con los obtenidos mediante conexiones a servicios WFS. Las funcionalidades SIG que implementa SignA son:

- Se admiten transformaciones entre los sistemas de referencia oficiales tanto en geográficas como en proyectadas en los diferentes usos.

- Visualización de las tablas de datos con todos los objetos geográficos y sus atributos y su posible exportación en formato Excel, GML y shapefile.

- Consulta de atributos o semánticas, filtrando los objetos geográficos que cumplen ciertos valores de atributo. Por ejemplo: mostrar el municipio de nombre igual Robledo de Chavela. 
- Consultas espaciales, utilizando varias clases de objeto geográfico y los operadores espaciales “contiene, se solapa, está incluido, etc.”, por ejemplo: mostrar los afluentes del Río Jarama.

- Áreas de influencia de un objeto geográfico. Por ejemplo: municipios en un entorno de $10 \mathrm{~km}$ de la central nuclear de nombre Santa María de Garoña.

- Descarga de los objetos geográficos resultantes de una consulta en formato GML o shapefile.

- Cálculo de perfiles longitudinales.

- Cálculo de rutas.

Las mejoras de usabilidad llevadas a cabo en las funcionalidades SIG han sido las siguientes:

- Asignar un nombre por defecto a la consulta en función de la capa a consultar.

- En la versión 3 se crearon una serie de capas con el resultado de una serie de consultas que frecuentemente se realizan al Instituto Geográfico Nacional, como los puntos extremos de España, los municipios más poblados, los centroides de municipios, provincias y comunidades autónomas, los puntos más altos y bajos de cada provincia, los picos más altos de cada cordillera, etc.

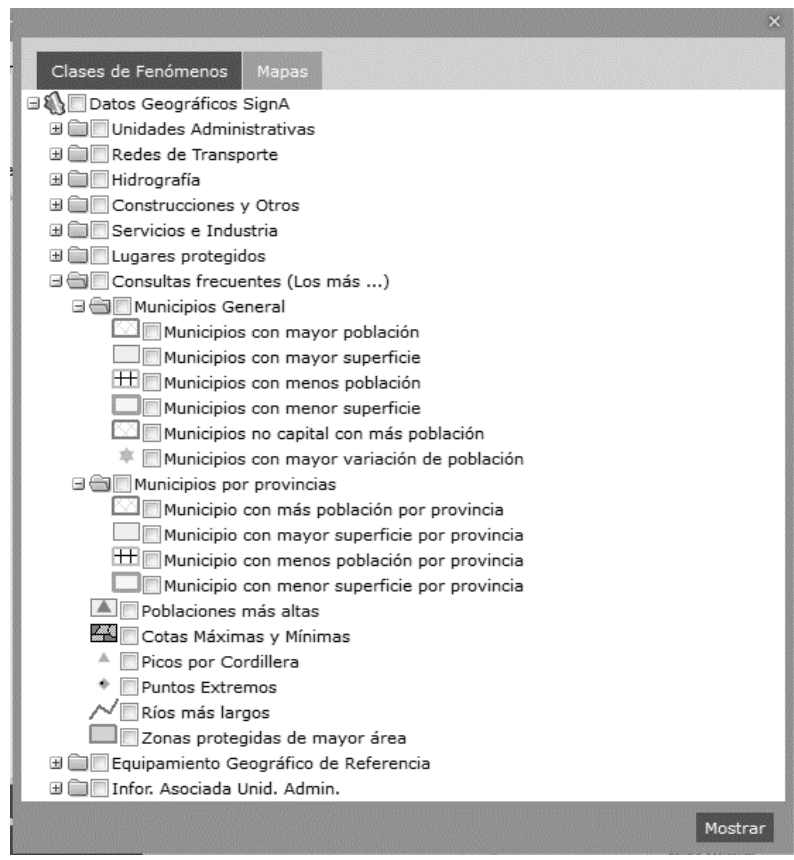

Figura 7. Capas con las consultas frecuentes. 
- En la versión 4 se han creado las consultas parametrizadas, ya configuradas para que los usuarios básicos sólo tengan que elegir uno o dos parámetros. Por ejemplo, para hacer una consulta de los afluentes de un río, en vez de hacer una consulta de los ríos que tocan al río de nombre $x$, el usuario únicamente tiene que elegir el nombre del río de una lista de valores propuestos. Otros ejemplos de consultas rápidas son: área de influencia de $x$ metros alrededor de una entidad de población $y$, cuenca hidrográfica de un río $x$, municipios por los que discurre un río $x$, alojamientos de ocio a $x$ metros de una entidad de población $y$, etc.
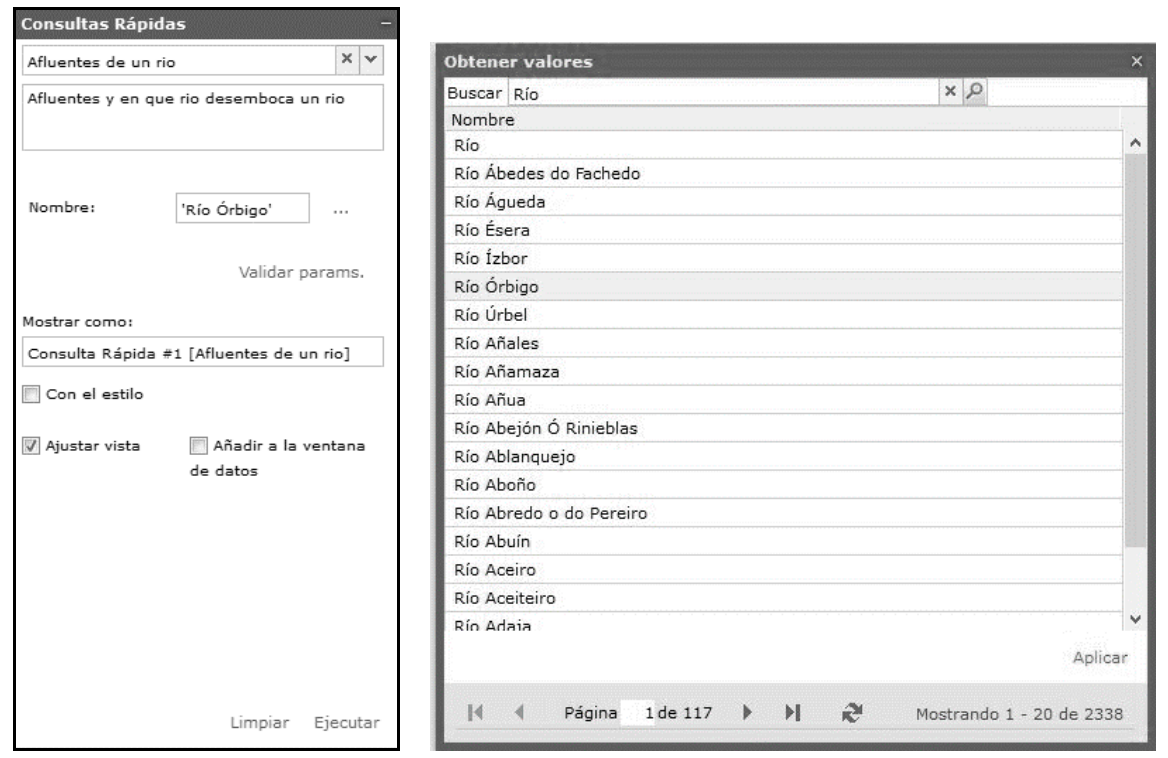

Figura 8. Ejemplo de consulta rápida “afluentes de un río”.

- En la versión 4, en las consultas se han añadido también las opciones de ajustar vista al resultado de la consulta y de añadir resultado a la vista de datos. Hasta ahora había que hacer zoom a la zona y añadir los datos manualmente.

- Otra de las mejoras que se hicieron en la versión 3 es la descarga y carga del entorno de trabajo en un fichero GPW con un solo click. Este fichero almacena todas las conexiones y las consultas, de manera que se puede enviar a otro usuario o cargar en otro ordenador para seguir trabajando. En versiones anteriores la opción de descarga requería de varios pasos y muy complejos: había que añadir el espacio de trabajo y luego una vez creado descargarlo. A partir de la versión 3 basta con pinchar sobre los iconos correspondientes (disquete). 


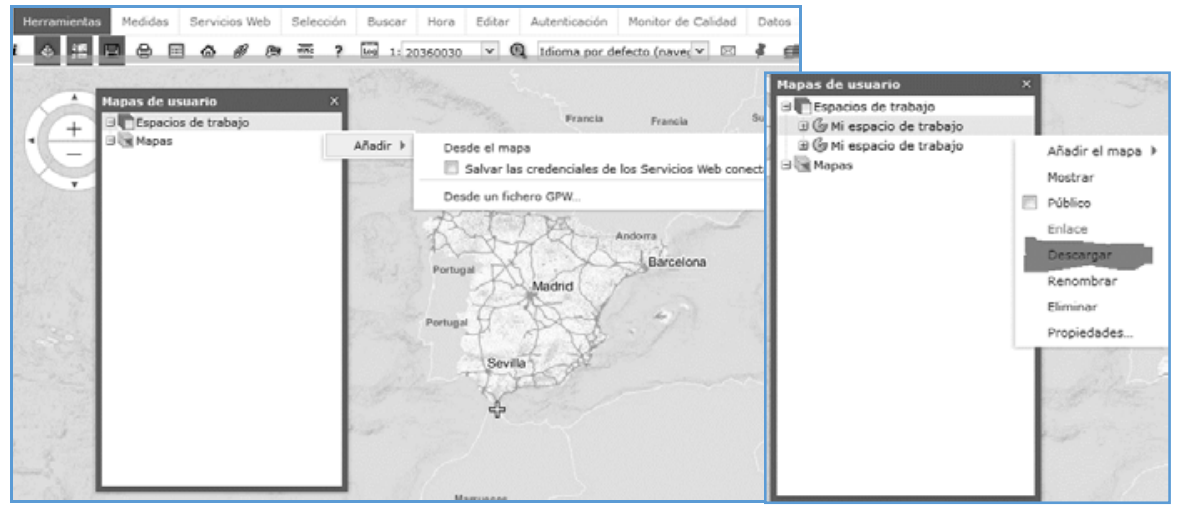

Figura 9. Descarga de espacio de trabajo en versiones 1 y 2.

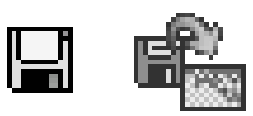

Figura 10. Carga y descarga de espacio de trabajo en versiones 3 y 4 con un solo click.

- Los usuarios que deseen descargar los datos brutos pueden pulsar en el ícono de descarga y el sistema les redirige directamente al Centro de Descargas del CNIG con el mismo ámbito geográfico que tuviesen en el SignA.
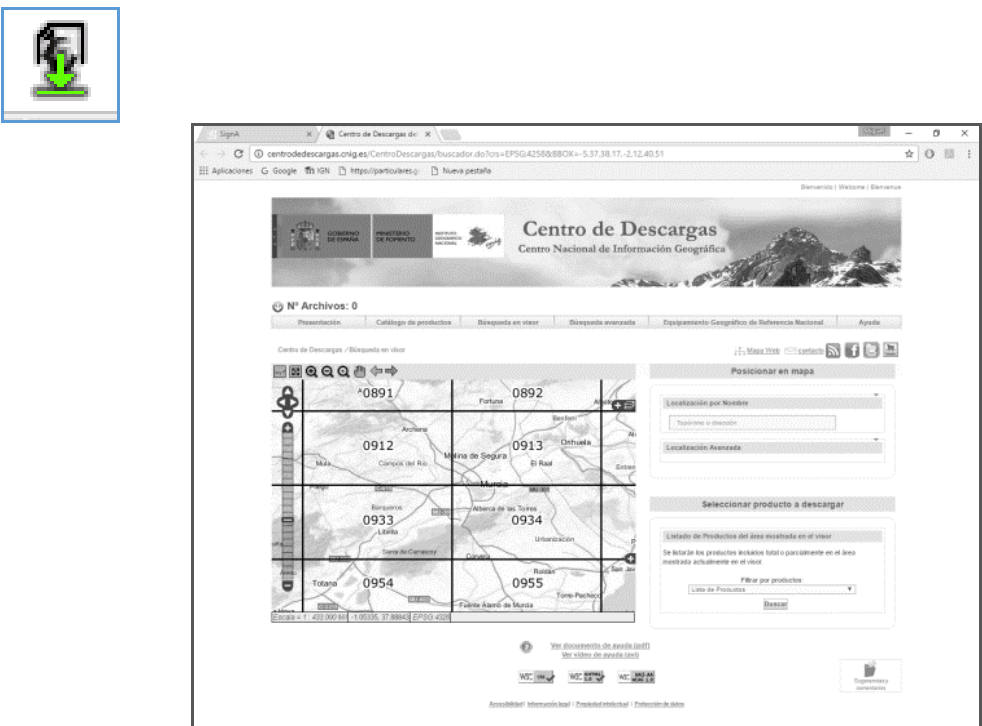

Figuras 11. Acceso directo al Centro de Descargas del CNIG por ámbito geográfico. 


\section{Estudios de usabilidad}

Después del lanzamiento de la primera versión se hicieron estudios y trabajos encaminados a mejorar la usabilidad del geoportal. Es un tema clave para el éxito del proyecto y todo un reto el lograr conjugar con éxito el mundo de los SIG y el mundo de las IDE en un mismo entorno. Estas son disciplinas de la geomática que requieren una amplia base de conocimientos, lo que hace que las herramientas sean complejas.

Persiguiendo este fin, se hicieron dos estudios de usabilidad cuyas conclusiones se resumen a lo largo de este epígrafe. Estos estudios son:

- Estudio sobre la usabilidad del visualizador del Sistema de Información Geográfica Nacional (Real Sociedad Geográfica de España, 2015).

- Aplicación del Diseño Centrado en el Usuario para mejorar un Geoportal, Universidad Politécnica de Madrid (Marín y Riesco, 2012).

En ambos estudios se detectaron una serie de problemas (también multitud de virtudes) que se están tratando de resolver en las sucesivas versiones que se han ido desarrollando del geoportal. Los principales problemas encontrados fueron:

- Velocidad de carga y de respuesta de algunos servicios.

- Errores y excepciones de la aplicación a veces no notificadas o notificación no clara.

- Usuarios no entienden el valor añadido que aporta SignA frente a otros visualizadores.

- Mejorar buscador en la ayuda.

En respuesta a estos problemas se han seguido estrategias para incluir en los nuevos desarrollos lo siguiente:

- Velocidad:

- Sustitución, en la medida de lo posible, de los servicios WMS por servicios WMTS cuya mejora en el rendimiento, está valorada en ser unas 100 veces más rápido.

- Mejora del rendimiento durante la carga inicial del geoportal.

- Mejora del rendimiento de la petición GetFeatureInfo, operación de consulta de información sobre una capa.

- Mensajes de error:

- Introducción de nuevos mensajes de error y depuración de los existentes en el caso de que no sean mensajes claros y evocadores. 
- Adicción de mensaje "no encontrado" cuando las consultas a servicios de nomenclátor no devuelvan ningún registro.

- Mejora de la semántica de algunos cuadros de diálogo.

- Valor añadido de SignA:

- Enfoque más profesional. Se planean estrategias de divulgación entre sectores especializados como cursos de formación en Protección Civil de España o seminarios de formación en la Facultad de Geografía e Historia de la Universidad Complutense de Madrid (UCM).

- Estrategia de divulgación de las capacidades SIG e IDE de SignA a través de las redes sociales y plataformas online del IGN.

- Buscador de ayuda:

- Se ha corregido el buscador de la ayuda, para que siempre proporcione resultados, aunque no se ajusten de manera exacta a la sintaxis de la búsqueda.

Otras mejoras efectuadas tras el análisis, introducidas por el equipo de SignA de la usabilidad y rendimiento del visualizador, han sido las siguientes:

- Mejora de la usabilidad de la interfaz que accede al servicio WPS de cálculo de rutas.

- Mejora de la funcionalidad que muestra la cota del punto al mover el cursor por la ventana de mapa.

- Actualizaciones para mejorar la usabilidad de la herramienta de impresión.

- Mejoras en la usabilidad la herramienta de guardar mapas de usuario.

- Se añade reborde en los resultados de las medidas de distancia y área, ya que no se leían correctamente.

- Se añade el comando «zoom al resultado de una consulta» una vez que se haya ejecutado y la notificación con un mensaje que la consulta se ha resuelto satisfactoriamente.

- Acceso a botones de ayuda directa desde comandos.

- Eliminación de espacios muertos.

- Simplificación la herramienta de edición para que no sea necesario introducir atributos (insertar entidades puntuales, línea les y superficiales).

- Añadir el botón de cerrar en el cuadro de perfiles longitudinales.

Utilizando la oportunidad que nos brinda este texto, se quiere invitar a todos los usuarios de SignA a remitir sus comentarios o sugerencias al equipo del proyecto y proponer mejoras en la usabilidad del geoportal a través del correo electrónico: signa@ign.es. 


\section{Futuro}

El proyecto SignA desempeña un papel privilegiado ya que es la herramienta preferente para consultar y explotar los datos y servicios geográficos del IGN en cualquiera de sus modalidades, aprovechando las posibilidades que ofrece el mundo IDE, orientado a estándares, y el mundo SIG, orientado a la eficiencia en el proceso.

Lo que se pretende es disponer de todos aquellos servicios estándar que se produzcan en el IGN, complementados con la funcionalidad que ofrece un SIG que pueda acceder a los datos. Por ello consideramos importante observar cómo nacen, avanzan y maduran los estándares OGC.

También se avanzará en la obtención de mejoras en el rendimiento de las consultas, para ello se pretende trabajar tanto en la base de datos, como en las aplicaciones y en la infraestructura informática. Especialmente en este último punto tenemos mucho que aprender y esperamos que el uso de la actual aplicación y la experiencia nos marquen las futuras necesidades de nuestra infraestructura informática.

Sin duda, la mejora en la usabilidad es una de las prioridades del proyecto ya que se trata de una herramienta muy potente y a la vez compleja. Es prioritario que tanto usuarios básicos como avanzados, entiendan las capacidades de la herramienta y sepan explotarla de acuerdo a sus necesidades.

Se está trabajando en la cuarta versión del geoportal SignA v.4, cuya publicación está prevista para junio de 2017, que traerá muchas novedades demandadas por los usuarios, como la carga de datos de usuario en diferentes formatos (Shape, GML, KML, GPX, DWG, etc.), con lo que SignA se convertirá en un caso de aplicación en la nube del tipo Software As a Service (SaaS), la creación de consultas sencillas mediante parámetros, la descarga de imágenes georreferenciadas y la descarga de datos en diferentes formatos.

\section{Conclusiones}

Con este artículo se pretende dar a conocer al usuario la forma actual de acceso a los datos del IGN, mostrando a SignA como un escaparate privilegiado de los productos y servicios del IGN; y por otra parte mostrar cómo el SignA representa el nodo de explotación SIG e IDE del IGN, es decir, un sitio web que mezcla de manera armónica e integrada los servicios OGC del IGN disponibles con la capacidad explotación SIG de las bases de datos del IGN.

Además, se conecta directamente a dicha base de datos, por lo que además de un nodo cliente de los servicios IDE del IGN es un Sistema de Información Geográfica que se puede explotar en remoto al estar accesible en la Red con un navegador.

En futuras versiones del visualizador se tenderá, todo lo que sea posible, hacia la mejora en la usabilidad y el uso de los estándares OGC, siempre y cuando existan 
soluciones eficientes y fiables de los requerimientos de los usuarios; en caso contrario, se complementará la explotación de servicios web con las funcionalidades proporcionadas por un software SIG.

\section{Bibliografía}

Directiva 2007/2/ce del Parlamento Europeo y del Consejo, de 14 de marzo de 2007, publicada en el Diario Oficial de la UE (DOUE) el 25 de abril de 2007, INSPIRE (Infraestructure for Spatial Information in Europe).

Ley 14/2010, de 5 de julio, sobre las infraestructuras y los servicios de información geográfica en España (LISIGE).

Marín López-Pastor, J. y Riesco García, L. (2012). “Aplicación del diseño centrado en el usuario para mejorar un Geoportal”, Universidad Politécnica de Madrid.

Sevilla, C.; Abad, P. y Villalón M. (2011). "Funcionalidades IDE del Geoportal SignA del IGN-CNIG".

Sevilla, C.; Potti, H.; Abad, P. y Rodríguez, A. (2010). SIGNA: Sistema de Información Geográfica Nacional. La puerta de acceso al Nodo IDE del IGN.

Sevilla Sánchez, C.; Villalón Esquinas, M.; Potti Manjavacas, H. y Sánchez Fanjul, J., (2016). "SignA un geoportal al alcance de los usuarios para la visualización, consulta y análisis SIG de los datos del IGN", Congreso Nacional de Topografía y Cartografía, TOPCART 2016, Toledo, España.

Sevilla Sánchez, C.; Villalón Esquinas, M. y Sánchez Fanjul, J. (2016). “Geoportal SignA v.3.0 del IGN-CNIG”, Revista Mapping, vol. 25, no. 175.

Real Sociedad Geográfica (2015). "Estudio elaborado por la Real sociedad Geográfica sobre la usabilidad del visualizador del sistema de Información Geográfica Nacional (SignA)", Madrid. 